\title{
Risk factors for and consequences of persistent lower respiratory symptoms among World Trade Center Health Registrants 10 years after the disaster
}

\author{
Stephen M Friedman, ${ }^{1}$ Mark R Farfel, ${ }^{1}$ Carey Maslow, ${ }^{1}$ Hannah T Jordan, ${ }^{1}$ Jiehui Li, ${ }^{1}$ \\ Howard Alper, ${ }^{1}$ James E Cone, ${ }^{1}$ Steven D Stellman, ${ }^{2}$ Robert M Brackbill ${ }^{1}$
}

\begin{abstract}
- Additional material is published online only. To view please visit the journal online (http://dx.doi.org/10.1136/ oemed-2015-103512).

${ }^{1}$ New York City Department of Health and Mental Hygiene, World Trade Center Health Registry, Long Island City, New York, USA

${ }^{2}$ Department of Epidemiology, Mailman School of Public Health, Columbia University, New York, New York, USA
\end{abstract}

\section{Correspondence to} Dr. Stephen M. Friedman, New York City Department of Health and Mental Hygiene, World Trade Center Health Registry, 125 Worth Street, New York, NY 10013, USA; Sfriedm2@health.nyc.gov

Received 16 December 2015 Revised 10 June 2016 Accepted 19 June 2016 Published Online First 22 July 2016

\section{CrossMark}

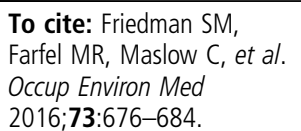

\section{ABSTRACT}

Objectives The prevalence of persistent lower respiratory symptoms (LRS) among rescue/recovery workers, local area workers, residents and passers-by in the World Trade Center Health Registry (WTCHR) was analysed to identify associated factors and to measure its effect on quality of life (QoL) 10 years after 9/11/ 2001.

Methods This cross-sectional study included 18913 adults who completed 3 WTCHR surveys (2003-2004 (Wave 1 (W1)), 2006-2007 (Wave 2 (W2)) and 20112012 (Wave 3 (W3)). LRS were defined as self-reported cough, wheeze, dyspnoea or inhaler use in the 30 days before survey. The prevalence of three LRS outcomes: LRS at W1; LRS at W1 and W2; and LRS at W1, W2 and W3 (persistent LRS) was compared with no LRS on WTC exposure and probable mental health conditions determined by standard screening tests. Diminished physical and mental health QoL measures were examined as potential LRS outcomes, using multivariable logistic and Poisson regression.

Results of the 4 outcomes, persistent LRS was reported by $14.7 \%$. Adjusted ORs for disaster exposure, probable post-traumatic stress disorder (PTSD) at W2, lacking college education and obesity were incrementally higher moving from LRS at W1, LRS at W1 and W2 to persistent LRS. Half of those with persistent LRS were comorbid for probable PTSD, depression or generalised anxiety disorder. Enrollees with persistent LRS were 3 times more likely to report poor physical health and $\sim 50 \%$ more likely to report poor mental health than the no LRS group.

Conclusions LRS, accompanied by mental health conditions and decreased QoL, have persisted for at least 10 years after 9/11/2001. Affected adults require continuing surveillance and treatment.

\section{BACKGROUND}

New onset lower respiratory symptoms (LRS) were reported by people directly exposed to the World Trade Center (WTC) attack in the weeks following 11 September 2001. ${ }^{1}$ Although the prevalence of frequent cough in firefighters fell from $54 \%$ in the first year after $9 / 11$ to $17 \%$ in year 2 , the prevalence of dyspnoea and wheeze remained elevated through 4 years of follow-up. ${ }^{2}$ Other rescue/recovery workers, local area workers and residents also experienced a high prevalence of persistent LRS $^{3-6}$ in the first 5 years after 9/11. Reibman et $a l^{7}$ described cough (46\%), wheeze (27\%) and dyspnoea on exertion (67\%) in local area workers and residents, clean-up workers and rescue/recovery workers seeking treatment up to May 2008. The prevalence of these symptoms was similar within each of these distinct exposure groups. The risk of persistent symptoms over the first 4 years post-9/11 in firefighters has been associated with earlier arrival time and longer duration at the site ${ }^{2}$ and in residents and local area workers, with greater exposure to settled dust at home or at work. ${ }^{8}$ Weakley et $a l^{9}$ reported stabilisation of the elevated prevalence of dyspnoea and wheeze among firefighters studied 5-9 years after 9/11. Wisnivesky et al ${ }^{10}$ documented an asthma prevalence of $18.1 \%$ in other rescue/recovery workers after 9 years.

To date, few data are available to determine whether the prevalence of LRS among 9/11-exposed people has remained elevated beyond 2010. Yip et $a l^{11}$ reported an elevated cumulative incidence of obstructive airways disease proportional to exposure in emergency medical service workers at 12 years after 9/11. More than two-thirds of WTC Health Registry (WTCHR) enrollees who were diagnosed with asthma within 2 years of being exposed to $9 / 11$ reported poorly or very poorly controlled asthma as of $2010-2011,{ }^{12}$ but the prevalence of LRS in the overall Registry population has not been described since $2009 .^{4}$

Among all WTC disaster-exposed groups, an increased prevalence of persistent LRS has been associated with post-traumatic stress disorder (PTSD), depression and generalised anxiety disorder (GAD) even while adjusting for intensity of exposure to the disaster. ${ }^{10}{ }^{13-17}$ Luft et al ${ }^{16}$ studying police and non-traditional responders (clean-up workers, building trades, transportation workers, clergy and others) suggested that PTSD mediates the association between exposure and LRS. Kotov et $a l^{18}$ analysed longitudinal data on these workers to demonstrate that PTSD increased the likelihood of LRS onset and decreased the rate of LRS remission.

LRS, especially in combination with PTSD, have been associated with indicators of poor quality of life (QoL) in WTCHR rescue/recovery workers, local area workers, residents and passersby. ${ }^{15}{ }^{17}$ Reduced QoL in firefighters and EMS workers has been attributed to physical conditions (including lower respiratory disease) and mental health conditions (including PTSD, depression and GAD). ${ }^{19}$ 
The objectives of this study are to document the persistence of LRS through 2011-2012 among adult rescue/recovery workers, residents, local area workers and passers-by enrolled in the WTCHR, to identify factors, such as disaster exposure and comorbid mental health conditions, which may increase the likelihood of persistent symptoms, and to gauge the impact of LRS on QoL.

\section{METHODS}

The Registry comprises individuals who met criteria for exposure to the disaster on or after 9/11/2001. ${ }^{20}$ These included (A) rescue, recovery and clean-up workers at the WTC site for at least one shift between 11 September 2001 and 30 June 2002, (B) Manhattan residents whose home address was below Canal Street, (C) local area workers whose place of work was below Chambers Street; and (D) passers-by in the area below Chambers Street on 9/11. Individuals were solicited from lists of potentially exposed adults provided by employers and government agencies (list-identified) or enrolled through an outreach and media campaign (self-identified). A total of 68046 adults aged $\geq 18$ responded to the first Registry survey (Wave 1 or W1, 2003-2004), $46020(67.6 \%)$ of these responded to the second Registry survey (Wave 2 or W2, 2006-2007) and 42395 responded to the third survey (Wave 3 or W3).

\section{Ethical approval}

The Institutional Review Boards of the New York City Department of Health and Mental Hygiene and the Centers for Disease Control and Prevention approved the Registry protocols.

Figure 1 Study population flow diagram listing exclusions and resulting four study groups.

\section{Respiratory outcomes}

LRS at W1 were defined as reported post-9/11 onset of wheeze, shortness of breath or persistent cough. For W2 and W3, number of symptomatic days and use of a physician-prescribed inhaler were added to the questionnaire; LRS in these surveys were defined as wheeze, shortness of breath or persistent cough for at least 8 of the 30 days prior to answering the survey or using an inhaler in the preceding 30 days. $^{21}$ In W3, symptoms were further specified to exclude those related to a cold, influenza or seasonal allergies.

Eight outcomes reflect the combinations of the presence or absence of LRS at W1, W2 and W3. Four were the focus of study: no LRS $(-,-,-)$; LRS at W1 only $(+,-,-)$, that is, LRS that had abated by W2; LRS at W1 and W2 only $(+,+,-)$, that is, LRS that had abated by W3; and LRS at W1, W2 and W3 $(+,+,+)$, 'persistent LRS'. Persistence of LRS is defined as LRS at W1, and LRS for at least 8 days or inhaler use in the 30 days before W2 and W3 $(+,+,+)$. This duration and continuation of symptoms is likely to represent more severe illness. These four outcomes were chosen because they most clearly trace the trajectory of symptoms possibly attributable to the disaster, either resolving or continuing through W3. The remaining four outcomes entail intermittent symptoms or onset of symptoms 5-10 years post-9/11.

\section{Study sample}

The analysis was limited to the adults who participated in the $\mathrm{W} 1, \mathrm{~W} 2$ and W3 surveys ( $\mathrm{n}=35857$, figure 1$)$. In order to concentrate on illness more likely attributable to WTC disaster exposures, enrollees who reported a pre-9/11 history of LRS

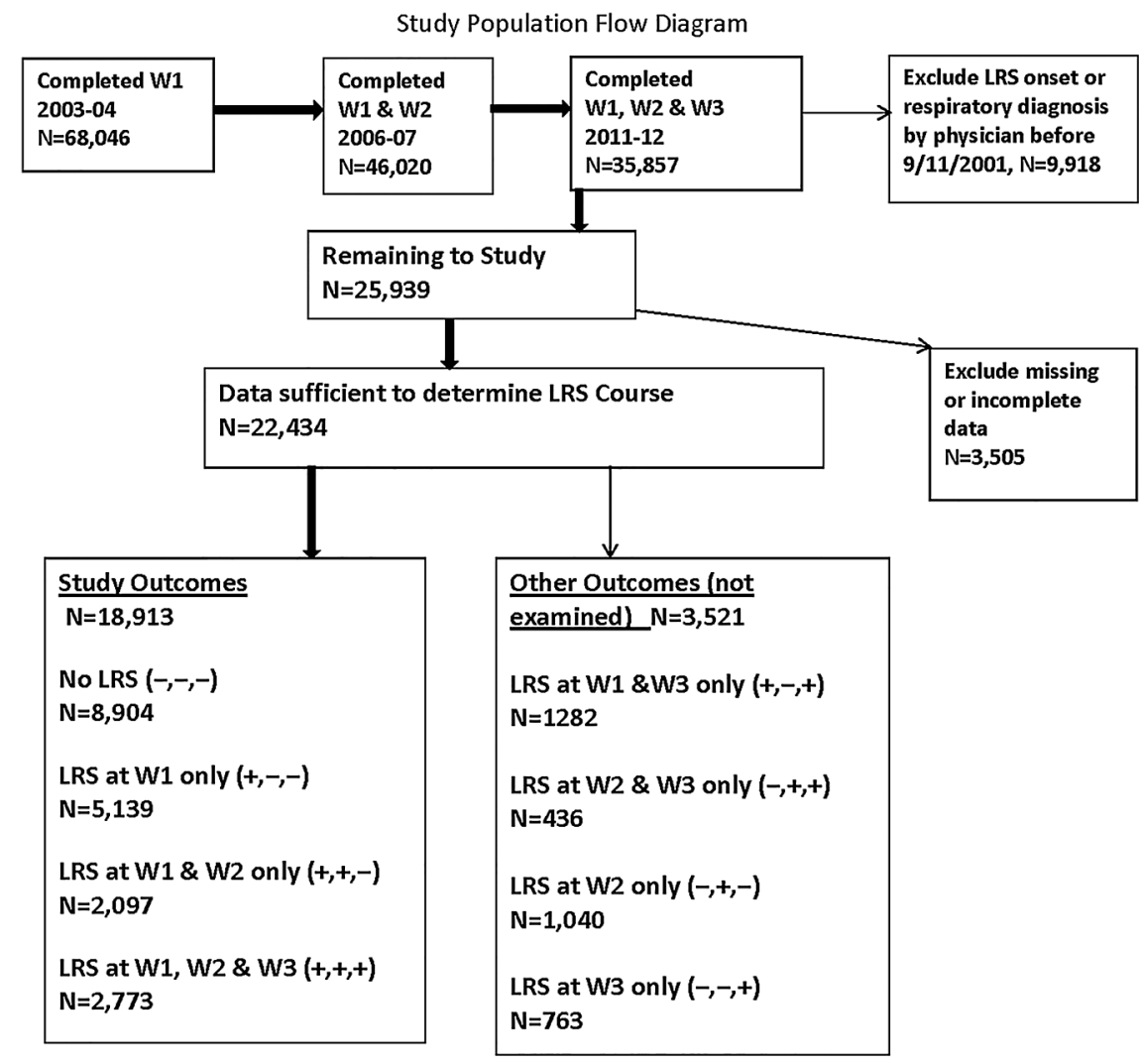


(3910) or pre-9/11 diagnosed chronic respiratory disease (asthma, reactive airways dysfunction syndrome, chronic bronchitis, emphysema, pulmonary fibrosis, sarcoidosis or other lung disease) (6008) were excluded, leaving 25 939. A conservative approach requiring consistency of response for the date of respiratory disease diagnosis across the three surveys was used in ascertaining the post-9/11 status. Symptom and inhaler use data sufficient to determine the trajectory of LRS were available for 22434 of 25939 (86.5\%). Of these, 18913 (84.3\%) were in the four groups, which are the focus of this report: $(-,-,-)$, $(+,-,-),(+,+,-)$ and $(+,+,+)$.

\section{Analysis variables}

Demographic variables included age on 9/11/2001, gender, racial/ethnic group and educational level at $\mathrm{W} 1$ and body mass index (BMI), smoking, marital status and social integration at W3. Social integration at W3 was based on the following criteria: having $>3$ close friends or relatives, and in the past 30 days, communicating with friends at least twice, attending a religious service at least twice, and being actively involved in a volunteer organization or club. Having each of these relationships or activities was counted as one point, and the categories were summed. A total of $0-1$ was considered low social integration, 2 was medium and 3-4 was high. ${ }^{22}$ Exposure was measured using a general matrix of WTC-associated physically and mentally challenging experiences. ${ }^{22}$ Since a combination of physical and psychological trauma exposures has driven the co-occurrence of respiratory symptoms and mental health problems, a scale that encompasses the full range of 9/11 exposures was used. This scale is a summation of the following 12 items each scored as 1 (present) or 0 (absent): (1) being in the North or South WTC towers or another building that collapsed in the attack; (2) witnessing three or more events (seeing planes hit the buildings, buildings collapsing, people falling or jumping from buildings, people injured or people running); (3) fear of being injured or killed; (4) having a relative killed on $9 / 11$; (5) having a friend killed on $9 / 11$; (6) having a coworker killed on $9 / 11$; (7) intense dust cloud exposure; (8) having lost or damaged possessions; (9) sustaining an injury other than eye irritation or injury; (10) being a rescue/recovery or clean-up worker; (11) evacuating one's home for at least 24 hours after 9/11; and (12) losing one's job because of 9/11. Exposure was classified as low (0-1), medium (2-3), high (4-5) and very high ( $\geq 6$ experiences).

The classification of probable PTSD 5-6 years after 9/11 was based on the score on the PTSD checklist (PCL) specific for the WTC disaster included in W2. The PCL is a 17-item selfreported instrument based on Diagnostic and Statistical Manual of Mental Disorders, fourth edition (DSM-IV) (American Psychiatric Association, 1994) criteria. Each item was scored on a five-point scale; a score of $\geq 44$ was considered probable PTSD. Measures of probable mental illness at W3, 10-11 years after 9/11, included (A) score $\geq 44$ on that PCL; (B) patient health questionnaire (PHQ8) score $\geq 10$ suggestive of depression; ${ }^{23}$ and (C) GAD7 test score $\geq 10$ consistent with an anxiety disorder. ${ }^{24}$ To summarise the overall effect of these three conditions, the number of positive test results was summed (0-3).

Standardised questions to measure QoL included general satisfaction with one's life and number of days in the past 30 days that physical or mental health was fair or poor, dichotomised as $<14$ and $\geq 14$ days. ${ }^{25}$

\section{Statistical analysis}

Analyses used SAS Statistical software, V.9.2. (SAS Institute, Cary, North Carolina, USA). Two-tailed tests were used in all analyses. The $\chi^{2}$ test was used to determine group differences on categorical variables. When the independent variable was categorical and ordered (educational level, BMI, current smoking level, degree of social interaction and level of exposure to the disaster), the effect on the prevalence of persistent LRS versus the remaining three LRS categories was compared for trend using the Cochran-Armitage trend test. A maximum $\mathrm{p}$ value of 0.05 was chosen for statistical significance.

Enrollees with persistent LRS were compared to those without LRS $(-,-,-)$ and to those whose LRS has abated $((+,-$, $-)$ and $(+,+,-)$ ). In bivariate analyses, the onset of LRS at W1, continuation of LRS at W2 and continuation of LRS at W3 were compared to never having LRS for key demographic variables and covariates shown to be significant in Registry data. These included cigarette smoking at W3, lack of health insurance, survey mode at W3 (mail, internet or computer-assisted telephone interview) and source of enrolment into the Registry (list-identified or self-identified). Lower rates of illness have been repeatedly found in list-identified enrollees compared with self-identified enrollees. ${ }^{4}$

The effect of WTC exposure variables and probable PTSD measured at W2 on the three outcomes with LRS compared to no LRS was examined while adjusting for demographics and covariates significant in the bivariate analysis in a multinomial logistic regression model assessed by odds ratios (ORs) with 95\% confidence intervals (CIs). Separate comparisons were also made between the LRS at W1 and W2 but not W3 group $(+,+,-)$ and the W1, W2 and W3 $(+,+,+)$ group in order to directly examine a factor's effect on persistence.

To assess the effect of comorbid psychological illness on persistence of LRS, prevalence ratios for the presence of LRS compared to no LRS, adjusted for demographics and exposure, were calculated using the Poisson distribution regression. ${ }^{26}$ In addition, the proportion of enrollees with a probable mental health condition was compared across the four LRS groups.

The effect of LRS on the prevalence of diminished $\mathrm{QoL}$ at W3 was examined in a Poisson distribution regression adjusting for demographics, covariates and mental health screening test results. ${ }^{26}$

\section{RESULTS}

The prevalence of persistent LRS $(+,+,+)$ was $12.4 \%$ among the 22434 enrollees with data sufficient to determine their LRS trajectory and $14.7 \%$ among the four groups (18 913) under study. The mean age of the 18913 enrollees was 42.2 years (median 42, IQR 34-50) at 9/11. The sample was $74 \%$ non-Hispanic white, $63 \%$ men, $89 \%$ non-smoking at W3, 57\% college graduates and $48 \%$ were rescue/recovery workers. The distribution of factors associated with persistent LRS or abated LRS $(+,+,-),(+,-,-)$ versus no LRS $(-,-,-)$ are presented in table 1. Bivariate analysis of demographics, exposure levels and other factors showed that the following were associated with an increased prevalence of persistent LRS: rescue/recovery work, age 45-64 years, male gender, black non-Hispanic or Hispanic racial ethnic group, current cigarette smoking, lacking a college education, lower level of social integration, lacking health insurance, intense exposure to the disaster and probable PTSD at W2. When the prevalence of persistent LRS was compared to the remaining LRS categories, the Cochran-Armitage trend test was significant at $\mathrm{p}<0.0001$ for increasing age group, cigarette smoking and exposure to the disaster and for decreasing educational level and social integration, and health insurance coverage. 
Table 1 Prevalence of LRS course by demographic factors, insurance, exposure to the disaster and mental health conditions; bivariate analysis*, $\mathrm{N}=18913$

\begin{tabular}{|c|c|c|c|c|c|}
\hline \multirow[b]{2}{*}{ Factor } & \multicolumn{5}{|c|}{ LRS course } \\
\hline & 18913 & $\begin{array}{l}\text { No LRS } \\
-,-,- \\
\text { Per cent } \\
47.1\end{array}$ & $\begin{array}{l}\text { LRS at W1 only } \\
+,-,- \\
\text { Per cent } \\
27.2\end{array}$ & $\begin{array}{l}\text { LRS at W1 and W2 only } \\
+,+,- \\
\text { Per cent } \\
11.1\end{array}$ & $\begin{array}{l}\text { LRS at W1, W2 and W3 } \\
+,+,+ \\
\text { Per cent } \\
14.7\end{array}$ \\
\hline \multicolumn{6}{|l|}{ Gender } \\
\hline Male & 11903 & 45.7 & 27.0 & 11.4 & 15.9 \\
\hline Female & 7010 & 49.4 & 27.5 & 10.6 & 12.5 \\
\hline \multicolumn{6}{|l|}{ Age category at 9/11/2001 } \\
\hline $18-24$ & 885 & 59.2 & 26.9 & 6.1 & 7.8 \\
\hline $25-44$ & 10291 & 45.7 & 28.4 & 11.3 & 14.5 \\
\hline $45-64$ & 7264 & 47.2 & 25.7 & 11.3 & 15.8 \\
\hline$\geq 65$ & 473 & 51.6 & 23.0 & 11.8 & 13.5 \\
\hline \multicolumn{6}{|l|}{ Eligibility group } \\
\hline Rescue/Recovery workers & 8995 & 41.7 & 27.6 & 12.0 & 18.7 \\
\hline Residents & 2494 & 52.4 & 27.2 & 9.7 & 10.7 \\
\hline Area workers & 6591 & 52.2 & 26.6 & 10.4 & 10.7 \\
\hline Passersby & 816 & 48.9 & 27.0 & 10.5 & 13.6 \\
\hline \multicolumn{6}{|l|}{ Race/ethnicity } \\
\hline White non-Hispanic & 14070 & 50.3 & 26.4 & 10.0 & 13.2 \\
\hline Black non-Hispanic & 1626 & 37.6 & 31.0 & 15.3 & 16.2 \\
\hline Hispanic & 1821 & 32.6 & 28.0 & 14.8 & 24.6 \\
\hline Asian & 884 & 49.2 & 29.6 & 10.2 & 11.0 \\
\hline Other & 512 & 36.1 & 28.3 & 15.2 & 20.3 \\
\hline \multicolumn{6}{|l|}{ Education‡ } \\
\hline High school or less & 3617 & 35.9 & 26.0 & 14.2 & 23.8 \\
\hline Some college & 4433 & 37.6 & 28.4 & 13.6 & 20.4 \\
\hline College/post-graduate & 10780 & 54.7 & 27.1 & 9.0 & 9.2 \\
\hline \multicolumn{6}{|l|}{ BMI reported at W3 $\ddagger$} \\
\hline Normal $(<25)$ & 5688 & 55.7 & 26.4 & 8.6 & 9.4 \\
\hline Overweight (25-29) & 7486 & 47.3 & 27.9 & 10.8 & 14.0 \\
\hline Obese $(\geq 30)$ & 5433 & 37.8 & 27.0 & 14.1 & 21.1 \\
\hline \multicolumn{6}{|l|}{ Smoking at W3‡ } \\
\hline Every day & 1049 & 29.5 & 23.2 & 16.8 & 30.6 \\
\hline Some days & 709 & 36.2 & 29.2 & 14.1 & 20.5 \\
\hline Not at all & 16908 & 48.7 & 27.3 & 10.6 & 13.4 \\
\hline \multicolumn{6}{|l|}{ Social integration at W3‡ } \\
\hline Low & 2597 & 34.5 & 27.8 & 13.1 & 24.6 \\
\hline Medium & 7191 & 47.8 & 27.1 & 10.9 & 14.1 \\
\hline High & 8607 & 50.2 & 27.2 & 10.6 & 12.0 \\
\hline \multicolumn{6}{|l|}{ Lacking health insurance $\neq$} \\
\hline No & 16315 & 48.4 & 27.2 & 10.9 & 13.5 \\
\hline Some time since $9 / 11$ but not in past 12 months & 1363 & 41.7 & 26.9 & 11.1 & 20.4 \\
\hline Lacking in past 12 months & 917 & 34.6 & 27.6 & 13.3 & 24.5 \\
\hline \multicolumn{6}{|l|}{ Exposure scale $\ddagger$} \\
\hline Low & 4874 & 63.8 & 21.6 & 6.7 & 7.9 \\
\hline Medium & 7970 & 48.8 & 27.1 & 11.0 & 13.1 \\
\hline High & 4373 & 35.4 & 31.5 & 13.7 & 19.3 \\
\hline Very high & 1696 & 20.9 & 32.4 & 17.5 & 29.3 \\
\hline \multicolumn{6}{|l|}{ Probable PTSD at W2 } \\
\hline No & 15530 & 53.2 & 27.9 & 9.4 & 9.5 \\
\hline Yes & 2893 & 16.8 & 23.4 & 19.8 & 40.0 \\
\hline \multicolumn{6}{|l|}{ Probable PTSD at W3 } \\
\hline No & 15817 & 52.0 & 28.0 & 10.0 & 10.0 \\
\hline Yes & 2441 & 17.5 & 22.1 & 16.8 & 43.6 \\
\hline \multicolumn{6}{|l|}{ Probable depression at W3 } \\
\hline No & 15880 & 51.6 & 28.0 & 10.1 & 10.3 \\
\hline Yes & 2481 & 20.0 & 21.8 & 16.6 & 41.5 \\
\hline
\end{tabular}


Table 1 Continued

\begin{tabular}{|c|c|c|c|c|c|}
\hline \multirow[b]{2}{*}{ Factor } & \multicolumn{5}{|c|}{ LRS course } \\
\hline & 18913 & $\begin{array}{l}\text { No LRS } \\
-,-,- \\
\text { Per cent } \\
47.1\end{array}$ & $\begin{array}{l}\text { LRS at W1 only } \\
+,-,- \\
\text { Per cent } \\
27.2\end{array}$ & $\begin{array}{l}\text { LRS at W1 and W2 only } \\
+,+,- \\
\text { Per cent } \\
11.1\end{array}$ & $\begin{array}{l}\text { LRS at W1, W2 and W3 } \\
+,+,+ \\
\text { Per cent } \\
14.7\end{array}$ \\
\hline \multicolumn{6}{|c|}{ Probable GAD at W3 } \\
\hline No & 16466 & 50.3 & 27.8 & 10.4 & 11.5 \\
\hline Yes & 1951 & 21.9 & 21.4 & 16.4 & 40.3 \\
\hline
\end{tabular}

${ }^{*}$ All factors are significant by $\chi^{2}$ at $p<0.0001$.

†The total may not add to $100 \%$ due to rounding and may be $<18913$ due to missing data. $\neq$ Cochran-Armitage trend test for persistent LRS versus other three categories significant at $\mathrm{p}<0.0001$.

BMI, body mass index; GAD, generalised anxiety disorder; LRS, lower respiratory symptoms; PTSD, post-traumatic stress disorder; W1, Wave 1; W2, Wave 2; W3, Wave 3.

When these factors were considered in a multinomial logistic regression model, adjusted ORs (aORs) for these variables except race/ethnicity were incrementally higher for those with LRS at W1; LRS at W1 and W2; and LRS at W1, W2, and W3, than for those without LRS reported in any of the surveys (table 2). For example, for probable PTSD at W2, the aOR rose from $1.9(95 \%$ CI 1.7 to 2.2$)$ for LRS at W1 only to $7.6(95 \%$ CI 6.7 to 8.7 ) for persistent LRS. (A version of table 2 with all the risk factors in the model is available as online supplementary appendix table S1).

When enrollees with persistent LRS were compared to those with LRS at W1 and W2 but not W3, aORs for less than college education (aOR 1.4 (95\% CI 1.2 to 1.6$)$ ), obese BMI (aOR 1.3 (95\% CI 1.1 to 1.6)), lower level of social integration (aOR 1.3 (95\% CI 1.1 to 1.6)), lack of health insurance at some time since $9 / 11$ (aOR 1.4 (95\% CI 1.1 to 1.7$)$ ) and probable
PTSD at W2 (aOR 1.8 (95\% CI 1.6 to 2.1) were significant. The exposure level did not remain significant.

The prevalence of LRS over the three surveys was closely related to the presence of a probable mental health condition. Prevalence ratios, adjusted for demographic factors and other covariates, were incrementally and significantly elevated from LRS at W1 only to persistent LRS (figure 2). For example, for depression, the adjusted prevalence ratio (aPR) rose from 1.3 (95\% CI 1.2 to 1.4 ) for the group with LRS at W1 only to 2.4 (95\% CI 2.2 to 2.6) for the persistent LRS group when each was compared to the no LRS group. When compared with enrollees with LRS at W1 and W2 only, enrollees with persistent LRS also had significantly elevated aPRs for each mental health condition (for GAD aPR=1.3 (95\% CI 1.2 to 1.4), for depression $\mathrm{aPR}=1.4(95 \% \mathrm{CI} 1.3$ to 1.4$)$ and for PTSD $\mathrm{aPR}=1.4$ (95\% CI 1.3 to 1.5$)$.

Table 2 Multinomial logistic regression of factors associated with LRS course at W1, W2 and W3, N=17 316

\begin{tabular}{|c|c|c|c|}
\hline \multirow[b]{2}{*}{ Factor } & \multicolumn{3}{|c|}{ LRS course } \\
\hline & $\begin{array}{l}\text { LRS at W1 only vs no LRS } \\
\text { Adjusted ORs and } 95 \% \mathrm{Cls}^{*}\end{array}$ & $\begin{array}{l}\text { LRS at W1, W2 only vs no LRS } \\
\text { Adjusted ORs and } 95 \% \mathrm{Cls}^{*}\end{array}$ & $\begin{array}{l}\text { LRS at W1, W2, W3 vs no LRS } \\
\text { Adjusted ORs and } 95 \% \mathrm{Cls}^{*}\end{array}$ \\
\hline \multicolumn{4}{|l|}{ Exposure scale } \\
\hline Low & Ref & Ref & Ref \\
\hline Medium & $1.5(1.4$ to 1.6$)$ & $1.8(1.6$ to 2.1$)$ & $1.7(1.5$ to 2.0$)$ \\
\hline High & 2.3 (2.0 to 2.5$)$ & 2.8 (2.3 to 3.3$)$ & 2.9 (2.5 to 3.4$)$ \\
\hline Very high & 3.7 (3.1 to 4.3$)$ & $5.2(4.2$ to 6.4$)$ & $5.3(4.3$ to 6.5$)$ \\
\hline \multicolumn{4}{|l|}{ Probable PTSD at W2 } \\
\hline No & Ref & Ref & Ref \\
\hline Yes & $1.9(1.7$ to 2.2$)$ & 4.2 (3.6 to 4.9$)$ & 7.6 (6.7 to 8.7$)$ \\
\hline \multicolumn{4}{|l|}{ Lacking health insurance } \\
\hline No & Ref & Ref & Ref \\
\hline Some time since $9 / 11$ but not in past 12 months & $1.1(0.91$ to 1.2$)$ & 0.99 (0.80 to 1.2$)$ & $1.4(1.1$ to 1.6$)$ \\
\hline Lacking in past 12 months & $1.2(1.0$ to 1.5$)$ & $1.3(1.0$ to 1.7$)$ & 1.7 (1.4 to 2.1$)$ \\
\hline \multicolumn{4}{|l|}{ Smoking at W3 } \\
\hline Not at all & Ref & Ref & Ref \\
\hline Some days & $1.2(1.0$ to 1.5$)$ & $1.4(1.1$ to 1.8$)$ & $1.2(0.96$ to 1.6$)$ \\
\hline Every day & $1.2(0.99$ to 1.4$)$ & $1.9(1.5$ to 2.3$)$ & 2.3 (1.9 to 2.8$)$ \\
\hline \multicolumn{4}{|l|}{ BMI reported at W3 } \\
\hline Normal $(<25)$ & Ref & Ref & Ref \\
\hline Overweight (25-29) & $1.2(1.1$ to 1.3$)$ & $1.3(1.1$ to 1.5$)$ & 1.5 (1.3 to 1.7$)$ \\
\hline Obese $(\geq 30)$ & $1.3(1.2$ to 1.5$)$ & 1.9 (1.6 to 2.2$)$ & 2.4 (2.1 to 2.8 ) \\
\hline
\end{tabular}


Figure 2 Probable mental health conditions and LRS course. Each of the three groups with LRS is compared to the group with no symptoms as to the prevalence of each of the three probable mental health conditions (based on scores on standard screening tests). LRS, lower respiratory symptoms.

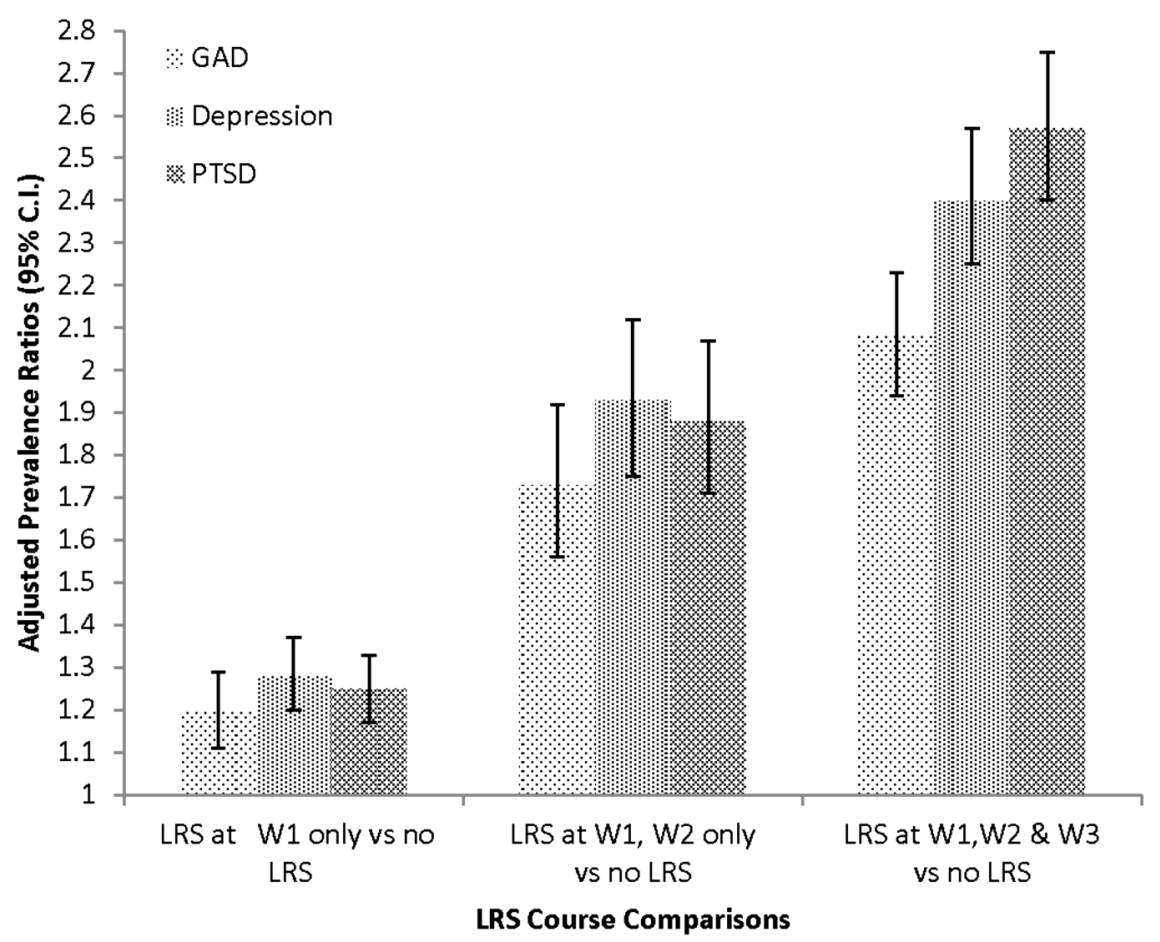

The proportion of enrollees with one, two or all three probable mental health conditions at W3 was calculated for each of the four LRS outcome groups (figure 3). Half (49.6\%) of the enrollees with persistent LRS had an elevated screening test result for at least one of these mental health conditions, and a third (35.2\%) had elevated scores for at least two conditions.

\section{QoL indicators}

In bivariate analyses, enrollees with persistent LRS were significantly more likely to have reported lower general satisfaction with life, and with regard to 30 days prior to $\mathrm{W} 3$, they were more likely to have reported at least 14 days of poor physical

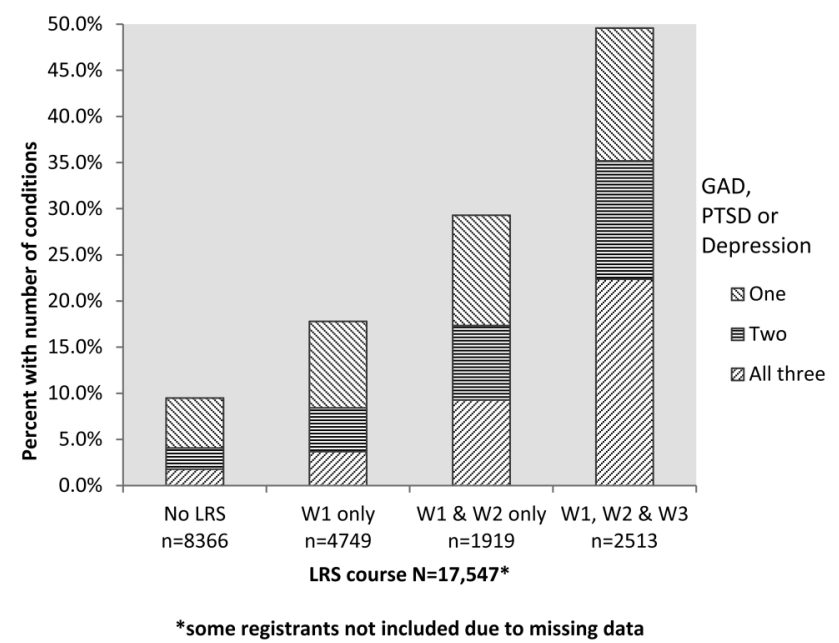

Figure 3 Proportional prevalence of count of probable mental health conditions at W3 by LRS course. The proportion of registrants with one, two or three positive screening test results is presented for each LRS group. LRS, lower respiratory symptoms; GAD, generalised anxiety disorder; PTSD, post-traumatic stress disorder. health and poor mental health than enrollees who had not reported LRS at any of the surveys $(p<0.0001$ for each indicator). Similarly, those with persistent LRS versus LRS at the first two but not the third survey showed a significantly higher prevalence of negative QoL indicators $(p<0.0001$ for all indicators).

The relationship between these three QoL indicators and LRS was examined while adjusting for the effects of demographics, BMI at Wave 3, exposure to the disaster and mental health conditions at Wave 3 (table 3). Even after adjusting for the count (0-3) of probable mental health conditions, the prevalence of a negative QoL outcome for each indicator was significantly higher among those with persistent LRS versus no LRS. The prevalence of reported poor physical health was more than three times higher in the group with persistent LRS $(\mathrm{aPR}=3.2,95 \%$ CI 2.9 to 3.6), and the prevalence of reported poor mental health was nearly $50 \%$ higher in this group $(\mathrm{aPR}=1.5,95 \%$ CI 1.3 to 1.6$)$. Furthermore, those with persistent LRS were significantly more likely than those with LRS at W1 and W2 only, to trend towards worse QoL indicators (aPRs between 1.1 and 1.8).

\section{DISCUSSION}

Cough, wheeze and dyspnoea, beginning after 9/11, have persisted among WTCHR enrollees for at least 10 years. Half of the enrollees with persistent LRS also had at least one mental health condition. Comorbid LRS and mental illness have been reported in rescue/recovery workers by other WTC disaster investigators $^{10141618}$ and in Registry surveys from 2003-2004 and 2006-2007 among workers and residents. ${ }^{4} 151727$ This study extends those findings to demonstrate that the co-occurrence of respiratory and mental health illness has continued for at least 10 years after the disaster and that reported persistent LRS were highly associated with probable GAD, depression and PTSD separately and in combination. This study did not attempt to look at the longitudinal occurrence of LRS and mental illness or to detail the interactions between them. 
Table 3 Adjusted prevalence ratios of LRS course predicting QoL at W3 survey*, N=16,615

\begin{tabular}{|c|c|c|c|c|c|c|c|}
\hline \multirow[b]{3}{*}{ LRS course } & \multicolumn{7}{|c|}{ QoL measures } \\
\hline & \multirow[b]{2}{*}{ Total } & \multicolumn{2}{|c|}{ Satisfaction with life } & \multicolumn{2}{|c|}{$\begin{array}{l}\text { Poor physical health in } \\
\text { the past } 30 \text { days }\end{array}$} & \multicolumn{2}{|c|}{$\begin{array}{l}\text { Poor mental health in the past } \\
30 \text { days }\end{array}$} \\
\hline & & $\begin{array}{l}\text { Fair or poor } \\
(\%)\end{array}$ & $\begin{array}{l}\text { Adjusted prevalence } \\
\text { ratio }\end{array}$ & $\begin{array}{l}\geq 14 \text { days } \\
(\%)\end{array}$ & $\begin{array}{l}\text { Adjusted prevalence } \\
\text { ratio }\end{array}$ & $\begin{array}{l}\geq 14 \text { days } \\
(\%)\end{array}$ & $\begin{array}{l}\text { Adjusted prevalence } \\
\text { ratio }\end{array}$ \\
\hline No LRS & 8904 & 8.1 & Ref & 6.3 & Ref & 10.0 & Ref \\
\hline LRS at W1 only & 5139 & 11.9 & $1.1(0.99$ to 1.2$)$ & 10.4 & $1.4(1.3$ to 1.6$)$ & 14.2 & 1.1 (0.99 to 1.2$)$ \\
\hline LRS at W1, W2 only & 2097 & 17.8 & $1.2(1.1$ to 1.4$)$ & 17.9 & $1.9(1.6$ to 2.2$)$ & 22.2 & $1.2(1.1$ to 1.3$)$ \\
\hline LRS at W1, W2 and W3 & 2773 & 30.4 & $1.3(1.2$ to 1.5$)$ & 39.1 & $3.2(2.9$ to 3.6$)$ & 41.5 & $1.5(1.3$ to 1.6$)$ \\
\hline $\begin{array}{l}\text { LRS at W1, W2 and W3 vs LRS at } \\
\text { W1, W2 only }\end{array}$ & & & $1.1(1.0$ to 1.2$)$ & & $1.8(1.6$ to 2.0$)$ & & $1.3(1.2$ to 1.4$)$ \\
\hline
\end{tabular}

However, the study did document that enrollees with reported probable PTSD and LRS at W2 were almost twice as likely to report LRS at W3, suggesting that this mental health condition predisposes to ongoing respiratory illness. Notably, the strength of association between elevated PTSD at W2 and persistent LRS was of greater magnitude than the association between the level of exposure to the disaster and persistent LRS. Furthermore, when the persistent LRS group was compared with those whose LRS abated after W2, probable PTSD at W2 remained significantly associated but the exposure level did not.

Studies to elucidate the biological linkages between PTSD and respiratory disease $\mathrm{e}^{28}$ and to assess the impact of comorbid PTSD on uncontrolled LRS $^{29}$ or asthma ${ }^{30}$ in WTC-exposed people are ongoing. Recognising the burden of comorbid mental health and LRS, researchers are evaluating treatment modalities specific for their co-occurrence. ${ }^{31}$

Comorbid LRS and mental illness have been associated with greater decreased QoL than LRS alone in rescue/recovery workers, local area workers and residents. ${ }^{15} 1719$ Reported persistent LRS in this study were significantly associated with $\geq 14$ days of poor physical and with $\geq 14$ days of poor mental health, even while controlling for the number of coexisting mental health conditions. In addition, those with persistent LRS at W3 had significantly worse QoL than those with LRS at only W1 and W2. This finding suggests that the continuing presence of LRS at W3 negatively affected mental as well as physical health.

The burden of persistent LRS likely extends beyond the enrollees studied here. Extrapolating the prevalence of symptoms to the estimated 400000 adults exposed to the WTC disaster, 25 000 experienced persistent LRS during the 12-month interval before $\mathrm{W} 3^{20} 32$ (see online supplementary appendix table S2). This generalisation, however, may be affected by study selection factors (see limitations below).

Researchers are evaluating the efficacy of treatment for persistent LRS. ${ }^{29}{ }^{30}$ Although the W3 survey did not gather data on treatment effectiveness, a follow-up questionnaire on the treatment of asthmatics still symptomatic as of the W3 survey was included as part of the recently concluded fourth Registry survey (2015-2016), whose findings will be reported in due course.

A strength of WTCHR surveys, in general, is the ongoing response of rescue/recovery workers, residents and area workers; more than half the original adult cohort completed the third survey. With this sizeable database and follow-up rate, the effect of demographics, exposure and mental health factors on the prevalence of LRS can be assessed. Evidence of strength of the association between risk factors and the prevalence of LRS is provided by the increasing adjusted ORs across the four study trajectories $(-,-,-)$ to $(+,-,-)$ to $(+,+,-)$ to $(+,+,+)$. Similarly, there is a dose-response relationship for scaled risk factors within each LRS category. For example, adjusted ORs for persistent LRS increased as the exposure scale increases from low to very high, suggesting that more severe exposure induced more prolonged illness. To date there is little other data on the effect of exposure on long-term persistence of symptoms although new incident, physician-diagnosed obstructive airway disease for at least 7 years post-9/11 is associated with WTC exposure among firefighters. ${ }^{33}$

This study has several limitations. LRS data are derived solely from enrollees' self-report in WTCHR surveys and are clinically unvalidated. However, WTC self-reported illness data have been found to be reasonably reliable in previous studies. In a nested case-control study of local area workers and residents in the Registry, enrollees with LRS at W1, W2 and at examination 68 years post-9/11 were significantly more likely to show abnormal lung function on spirometry and impulse oscillometry than were asymptomatic enrollees. ${ }^{21}$ These findings were confirmed in a follow-up study 5 years later. ${ }^{34}$

Another possible limitation is selection bias, in which respondents to all three surveys may have chosen to continue to participate because they perceived that they had greater exposure to the disaster and experienced more prolonged symptoms. However, the magnitude of the associations between disaster exposures and factors such as the prevalence of PTSD and LRS at W2 was similar among W3 participants versus enrollees who completed W1 and W2 but not W3 (W3 drop-outs). ${ }^{35}$ Demographic factors differentiating W3 participants and non-participants were adjusted for in the current study by multivariable regression analysis. Still, the differences must be acknowledged in generalising the study findings to the entire WTCHR population and to the overall exposed population.

This study did not longitudinally examine LRS and PTSD to determine whether one preceded and/or exacerbated the other. Studies to gauge their interrelationship over time are underway using data from the three WTCHR surveys and, in rescue/recovery workers, using real-time measurements. ${ }^{36}$

Smoking cigarettes everyday was associated with persistent LRS. Smoking has been identified as a risk factor for poor respiratory outcome in WTC rescue/recovery workers ${ }^{37}$ and residents and local area workers. ${ }^{15}$ A smoking cessation 
programme has been available to Fire Department of New York rescue/recovery workers since $2002 .^{38}$ Since 2008 , the Registry has made multiple efforts to reach enrollees who have reported smoking cigarettes ${ }^{39}$ directing adults to sources of free nicotine patch replacement kits and brief counselling. Because individuals with PTSD are less likely to stop smoking without assistance, ${ }^{39}$ the Registry has targeted its activities towards this group, and since 2013, offered them referrals to a clinical trial smoking cessation programme aimed at adults with symptoms of PTSD. ${ }^{40}$ These data underscore the need for further efforts to reduce cigarette smoking in 9/11-exposed adults with LRS, especially those with comorbid mental health problems.

\section{CONCLUSION}

LRS and associated mental illness, resulting in diminished QoL, continued to affect thousands of WTCHR enrollees more than 10 years after $9 / 11$. Studies are needed to track the future course of LRS and to quantify the interrelationship between LRS and PTSD. As the interval from disaster exposure to current illness grows, mental illness is the most strongly identified determinant of LRS persistence. The association of LRS with mental health symptoms merits clinical consideration; these comorbidly affected patients require ongoing evaluation and care.

\section{What this paper adds}

- Lower respiratory symptoms (LRS) in adults exposed to the World Trade Center disaster are well-documented, but factors associated with their persistence 10 years later have not yet been identified.

- LRS have persisted in more than $12 \%$ of adults responding to the World Trade Center Health Registry surveys.

- Probable mental health conditions (post-traumatic stress disorder, depression and generalised anxiety disorder) are highly associated with symptom persistence.

- LRS are associated with decreased quality of life as indicated by self-reported physical and mental health status.

- Continued surveillance and simultaneous treatment of respiratory and mental illness are needed for this population.

Acknowledgements The authors thank Drs Charon Gwynn, James Hadler and Lisa Gargano for their thoughtful review of the manuscript and also thank Monique Fairclough and David Wu for assistance with the figures.

Contributors SMF, MRF and RMB are responsible for planning, conduct and reporting of the work and are the guarantors of the overall content. CM, HTJ, HA, JL, JEC and SDS are also responsible for planning, conduct and reporting of the work.

Funding This publication was supported by Cooperative Agreement Number 5U50/ OH009739 from the National Institute for Occupational Safety and Health (NIOSH) of the Centers for Disease Control and Prevention (CDC); U50/ATU272750 from the Agency for Toxic Substances and Disease Registry (ATSDR), CDC, which included support from the National Center for Environmental Health, CDC; and by the New York City Department of Health and Mental Hygiene (NYC DOHMH). Its contents are solely the responsibility of the authors and do not necessarily represent the official views of NIOSH-CDC or the Department of Health and Human Services.

Competing interests None declared.

Ethics approval The Institutional Review Boards of the New York City Department of Health and Mental Hygiene and the Centers for Disease Control and Prevention approved the Registry protocols.

Provenance and peer review Not commissioned; externally peer reviewed.

\section{REFERENCES}

1 Perlman SE, Friedman S, Galea $\mathrm{S}$, et al. Short-term and medium-term health effects of 9/11. Lancet 2011;378:925-34.
2 Webber MP, Gustave J, Lee R, et al. Trends in respiratory symptoms of firefighters exposed to the World Trade Center disaster: 2001-2005. Environ Health Perspect 2009;117:975-80.

3 Herbert R, Moline J, Skloot G, et al. The World Trade Center disaster and the health of workers: five year assessment of a unique medical screening program. Environ Health Perspect 2006;114:1853-8.

4 Brackbill RM, Hadler JL, DiGrande L, et al. Asthma and posttraumatic stress symptoms 5 to 6 years following exposure to the World Trade Center terrorist attack. JAMA 2009;302:502-16.

5 Lin S, Jones R, Reibman J, et al. Lower respiratory symptoms among residents living near the World Trade Center two and four years after 9/11. Int J Occup Environ Health 2010;16:44-52.

6 Mauer MP, Cummings KR, Hoen R. Long-term respiratory symptoms in World Trade Center responders. Occup Med 2010;60:145-51.

7 Reibman J, Liu M, Cheng Q, et al. Characteristics of a residential and working community with diverse exposure to World Trade Center dust, gas and fumes. J Occ Environ Med 2009;51:534-41.

8 Maslow CB, Friedman SM, Pillai PS, et al. Chronic and acute exposures to the World Trade Center disaster and lower respiratory symptoms: area resident and workers. Am J Public Health 2012;102:1186-94.

9 Weakley J, Webber MP, Gustave J, et al. Trends in respiratory diagnoses and symptoms of firefighters exposed to the World Trade Center disaster: 2005-2010. Prev Med 2011;53:364-9.

10 Wisnivesky JP, Teitelbaum SL, Todd AC, et al. Persistence of multiple illnesses in World Trade Center rescue and recovery workers: a cohort study. Lancet 2011;378:888-97.

11 Yip J, Zeig-Owens R, Webber MP, et al. World Trade Center-related physical and mental health burden among New York City Fire Department emergency medical service workers. Occup Environ Med 2016:73:13-20.

12 Jordan HT, Stellman SD, Reibman J, et al. Factors associated with poor control of 9/11-related asthma 10-11 years after the 2001 World Trade Center terrorist attacks. J Asthma 2015;52:630-7.

13 Gross R, Neria Y, Tao XG, et al. Posttraumatic stress disorder and other psychological sequelae among World Trade Center clean up and recovery workers. Ann NY Acad Sci 2006;1071:495-9.

14 Niles JK, Webber MP, Gustave J, et al. Co-morbid trends in World Trade Center Cough syndrome and probable posttraumatic stress disorder in firefighters. Chest 2011;140:1146-54.

15 Nair HP, Ekenga CC, Cone JE, et al. Co-occurring lower respiratory symptoms and posttraumatic stress disorder 5 to 6 years after the World Trade Center terrorist attack. Am J Public Health 2012;102:1964-73.

16 Luft BJ, Moline J, Pietrzak RH, et al. Exposure, probable PTSD and lower respiratory illness among World Trade Center rescue, recovery and clean-up workers. Psychol Med 2012;42:1069-79.

17 Friedman SM, Farfel MR, Maslow CB, et al. Comorbid persistent lower respiratory symptoms and posttraumatic stress disorder $5-6$ years post- $9 / 11$ in responders enrolled in the World Trade Center Health Registry. Am J Ind Med 2013;56:1251-61.

18 Kotov R, Bromet EJ, Schecter C, et al. Posttraumatic stress disorder and the risk of respiratory problems in World Trade Center responders: Iongitudinal test of a pathway. Psychosom Med 2015;77:438-48.

19 Yip J, Zeig-Owens R, Hall CB, et al. Health conditions as mediators of the association between World Trade Center exposure and health-related quality of life in firefighters and EMS workers. J Occup Environ Med 2016;58:200-6.

20 Farfel M, DiGrande L, Brackbill R, et al. An overview of 9/11 experiences and respiratory and mental health conditions among World Trade Center Health Registry enrollees. J Urban Health 2008;85:880-909.

21 Friedman SM, Maslow CB, Reibman J, et al. Case-control study of lung function in World Trade Center Health Registry area residents and workers. Am J Respir Crit Care Med 2011;184:582-9.

22 Brackbill RM, Stellman SD, Perlman SE, et al. Mental health of those directly exposed to the World Trade Center disaster: unmet mental health care need, mental health treatment service use, and quality of life. Soc Sci Med 2013:81:110-14

23 Kroenke K, Spitzer RL, Williams JB. The PHQ-9: validity of a brief depression severity measure. J Gen Intern Med 2001;16:606-13.

24 Spitzer RL, Kroenke K, Williams JB, et al. A brief measure for assessing generalized anxiety disorder: the GAD-7. Arch Intern Med 2006;166:1092-7.

25 Zahran HS, Kobau R, Moriarty DG, et al. Health-related quality of life surveillance-United States, 1993-2002. MMWR Surveill Summ 2005;54:1-35.

26 Zou G. A modified Poisson regression approach to prospective studies with binary data. Am J Epidemiol 2004;159:702-6.

27 Li J, Brackbill RM, Stellman SD, et al. Gastroesophageal reflux symptoms and comorbid asthma and posttraumatic stress disorder following the 9/11 terrorist attacks on World Trade Center in New York City. Am J Gastroenterol 2011;106:1933-41.

28 Griffin GD, Charron D, Al-Daccak R. PTSD: revisiting adrenergics, glucocorticoids, immune system effects and homeostatsis. Clin Trans/ Immunol 2014;3:e27.

29 Reibman J. Uncontrolled lower respiratory symptoms in the WTC survivor program. World Trade Center Health Program Research Principal Investigators Meeting; New York City, 19-20 November 2014. 
30 Wisnivesky J, Markowitz S. Prognosis and determinants of asthma morbidity in WTC rescue and recovery workers. World Trade Center Health Program Research Principal Investigators Meeting; New York City, 19-20 November 2014.

31 Gonzalez A, Bromet EJ, Luft BJ. Mind body treatment for WTC responders with comorbid PTSD and respiratory illness. World Trade Center Health Program Research Principal Investigators Meeting; New York City, 19-20 November 2014.

32 Murphy J, Brackbill RM, Thalji L, et al. Measuring and maximizing coverage in the World Trade Center Health Registry. Stat Med 2007;26:1688-701.

33 Hall CB, Liu $X$, ZeigOwens $R$, et al. The duration of an exposure response gradient between incident obstructive airways disease and work at the World Trade Center site: 2001-2011. PLoS Curr Published Online First: 20 May 2015. doi:10.1371/ currents.dis.8a93e7682624698558a76a1fa8c5893f

34 Jordan HT, Miller-Archie SA, Friedman SM, et al. Longitudinal study of lung function and respiratory symptoms among lower Manhattan area community members exposed to the 9/11/2001 World Trade Center attacks. American Thoracic Society meeting; May 2015 .
35 Yu S, Brackbill RM, Stellman SD, et al. Evaluation of non-response bias in a cohort study of World Trade Center terrorist attack survivors. BMC Res Notes 2015;8:42.

36 Kotov R, Monka W, Ruggero C, et al. Mental and physical health of WTC responders outside the clinic. World Trade Center Research Principal Investigators Autumn-Winter Meeting; New York City, 18-19 November 2015.

37 Aldrich TK, Gustave J, Hall CB, et al. Lung function in rescue workers at the World Trade Center after 7 years. N Eng J Med 2010;362:1263-72.

38 Bars MP, Banauch Gl, Appel D, et al. "Tobacco free with FDNY," the New York City Fire Department World Trade Center tobacco cessation study. Chest 2006;129:979-87.

39 Welch AE, Jasek JP, Caramanica K, et al. Cigarette smoking and 9/11-related posttraumatic stress disorder among World Trade Center Health Registry enrollees, 2003-12. Prev Med 2015;73: 94-9.

40 Gonzalez A, Bromet E, Kotov R, et al. Enhanced smoking cessation intervention. World Trade Center Research Principal Investigators Spring-Summer Meeting; New York City, 7-8 May 2015. 